\title{
Optimization of Experimental Parameters and Specimen Geometry for PulsedLaser Atom-Probe Tomography of Copper
}

\author{
R. Prakash Kolli ${ }^{1,2}$ and Frederick Meisenkothen ${ }^{2}$
}

${ }^{1}$ Department of Materials Science and Engineering, University of Maryland, College Park, MD, USA

${ }^{2}$ Materials Measurement Science Division, National Institute of Standards and Technology, Gaithersburg, MD, USA

Copper $(\mathrm{Cu})$ is a commercially important material used in semiconductor devices and other nanostructured materials. The accurate characterization of nano-scale deposition layers, dopant distributions, and impurity concentrations in $\mathrm{Cu}$ is of fundamental importance for the continued development of nanostructured materials. One quantitative compositional analysis technique that has the requisite sub-nanometer spatial resolution and high spectral resolution required for this type of characterization is atom-probe tomography (APT) [1]. During pulsed-laser APT three of the adjustable experimental parameters that influence the mass resolution are the laser pulse energy, laser pulse frequency, and specimen base temperature. For example, a pulse energy that is too low may degrade the mass resolution, $(m / \Delta m)$, and increase the tail size after major peaks in some materials [2]. On the other hand, it is well known that too high a pulse energy can cause uncontrolled field evaporation due to overheating of the tip thereby degrading mass resolution and dramatically increasing the tail size. Additionally, it has been demonstrated that laser pulse frequency affects the mass resolution and tail size in some materials but not others [3]. It is also known that too high a specimen base temperature may degrade the mass resolution and increase the size of the tails in some materials [4]. Additionally, the specimen geometry affects mass resolution depending on the thermal properties of the material [5]. Little attention has been paid to date on the influence of experimental parameters and specimen geometry during pulsed-laser APT of $\mathrm{Cu}$ hence their effects are not well understood. Optimization of these parameters and geometry would facilitate high mass resolution and improved mass spectra quality during APT analysis.

Initial results on the influence of laser pulse energy between 40 and $80 \mathrm{pJ}$, laser pulse frequency between 100 and $500 \mathrm{kHz}$, and specimen base temperature between 20 and $80 \mathrm{~K}$ during pulsed-laser APT are presented using a $3^{3}$ full factorial experimental test matrix. Data acquisition was performed employing a Cameca Local Electrode Atom-Probe (LEAP ${ }^{\circledR}$ ) 4000X Si instrument with a $355 \mathrm{~nm}$ ultraviolet (UV) pulsed-laser under ultra-high vacuum (UHV) conditions of $\sim 1.2 \times 10^{-8} \mathrm{~Pa}\left(\sim 9 \times 10^{-11}\right.$ torr). The mass resolution at full-width at half-maximum (FWHM), $(m / \Delta m)_{F W H M}$, the voltage-derived tip radius, $r_{t i p}$, and the estimated shank half-angle, $\gamma$, were determined in the Cameca Integrated Visualization and Analysis $\left(\right.$ IVAS $^{\circledR}$ ) software. Normalized mass spectra, which were normalized to the number of counts at the mass-to-charge-state $(\mathrm{m} / \mathrm{n})$ ratio that has the maximum ion detection events at ${ }^{63} \mathrm{Cu}^{1+}$, were used to compare the mass spectra of selected individual experiments. The results indicate that the mean of the quantity $(m / \Delta m)_{F W H M}$ improves with decreasing pulse energy, exhibits an optimum at a $250 \mathrm{kHz}$ pulse frequency, and improves with decreasing base temperature, Figs. 1(a) - (c). The mass resolution also exhibits an improving trend with increasing specimen tip radius and increasing shank half-angle. The tail magnitude and length after the major single-charged state peaks increases with decreasing pulse energy. The pulse frequency and base temperature have limited effect on the tail size. Interaction effects between experimental parameters and specimen geometry are also discussed [6]. 
References:

1. T. F. Kelly and D. J. Larson, Annu. Rev. Mater. Res. 42, (2012) 1.

2. K. Oh-ishi et al, Ultramicrosc. 111, (2011) 715.

3. A. Cerezo et al, Ultramicrosc. 107, (2007) 720.

4. D. E. Perea et al, J. Solid State Chem. 181, (2008) 1642.

5. J. H. Bunton et al, Microsc. Microanal. 13, (2007) 418.

6. Certain commercial equipment, instruments, or materials are identified in this paper in order to specify the experimental procedure adequately. Such identification is not intended to imply recommendation or endorsement by the National Institute of Standards and Technology, nor is it intended to imply that the materials or equipment identified are necessarily the best available for the purpose.
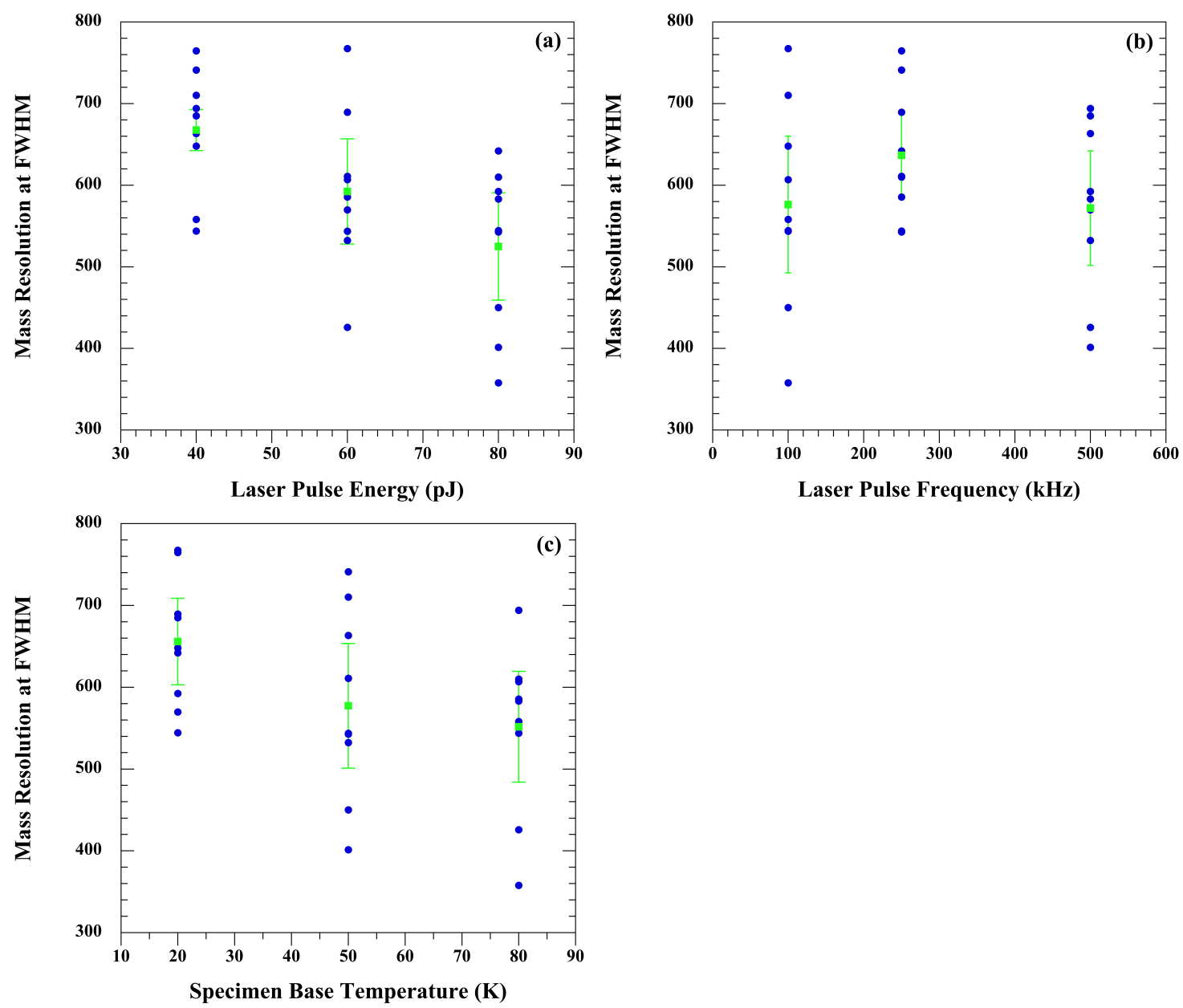

Figure 1. The effect of (a) laser pulse energy, (b) laser pulse frequency, and (c) the specimen base temperature on the quantity $(\mathrm{m} / \Delta m)_{F W H M}$ (blue dots) and its mean (green squares) for $\mathrm{Cu}$ during pulsed-laser APT is illustrated. The mean mass resolution at FWHM for $\mathrm{Cu}$ improves with decreasing pulse energy and base temperature. It exhibits an optimum at a $250 \mathrm{kHz}$ pulse frequency. 